LA-UR-99-3353

Approved for public release;

distribution is unlimited.

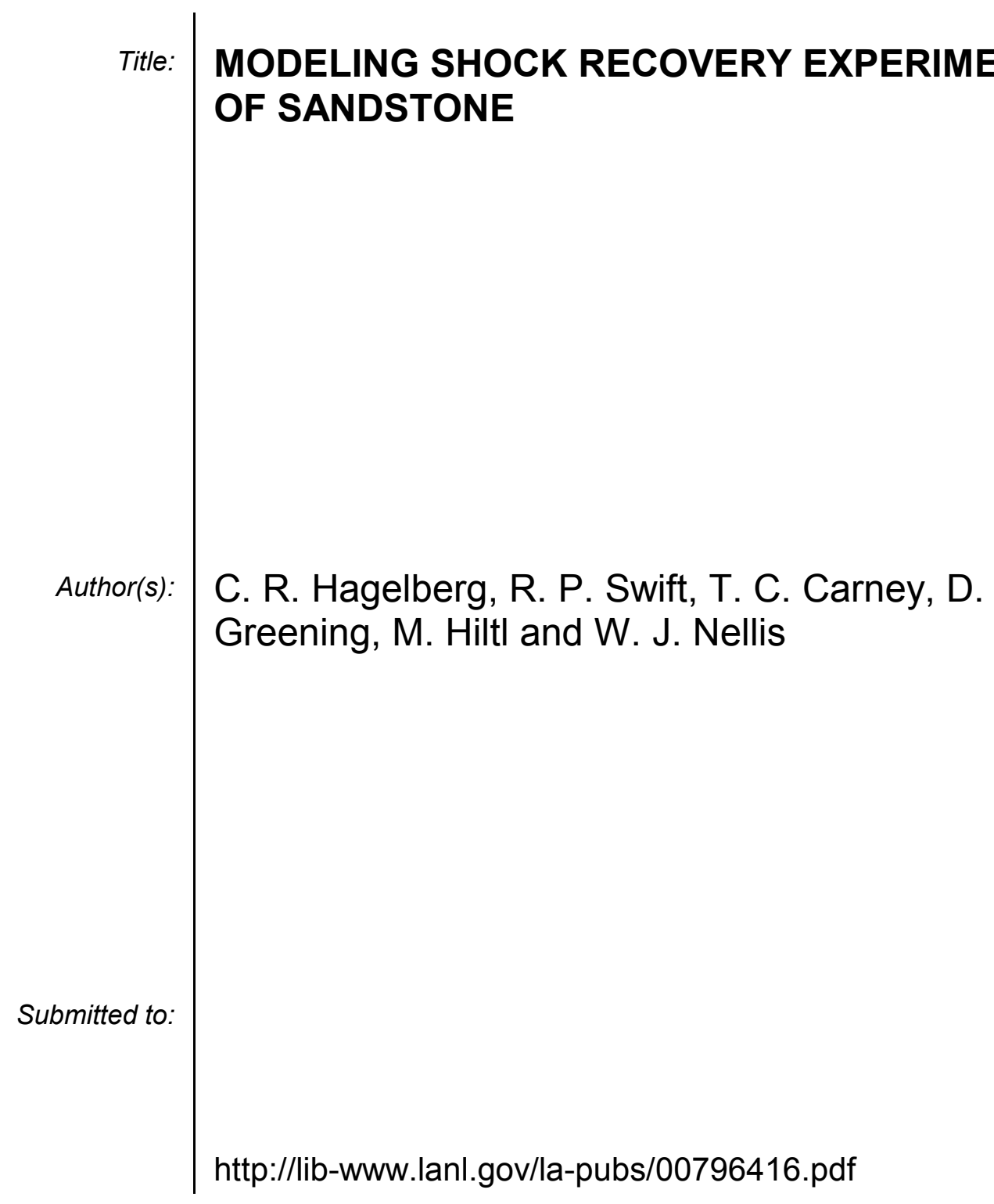

Los Alamos National Laboratory, an affirmative action/equal opportunity employer, is operated by the University of California for the U.S. Department of Energy under contract W-7405-ENG-36. By acceptance of this article, the publisher recognizes that the U.S. Government retains a nonexclusive, royaltyfree license to publish or reproduce the published form of this contribution, or to allow others to do so, for U.S. Government purposes. Los Alamos National Laboratory requests that the publisher identify this article as work performed under the auspices of the U.S. Department of Energy. Los Alamos National Laboratory strongly supports academic freedom and a researcher's right to publish; as an institution, however, the Laboratory does not endorse the viewpoint of a publication or guarantee its technical correctness. 


\title{
MODELING SHOCK RECOVERY EXPERIMENTS OF SANDSTONE
}

\author{
C. R. Hagelberg ${ }^{1}$, R. P. Swift ${ }^{1}$, T. C. Carney ${ }^{1}$, D. Greening ${ }^{1}$, M. Hiltt ${ }^{2}$, W. J. Nellis ${ }^{2}$ \\ ${ }^{1}$ Los Alamos National Laboratory \\ ${ }^{2}$ Lawrence Livermore National Laboratory
}

\begin{abstract}
We present results from mesoscale modeling of shock recovery experiments on Berea sandstone with the Smooth Particle Hydrodynamics and the Discrete Element methods. Each grain is represented with clusters of Discrete Element particles to provide explicit representation of the grain and pore structure. The grain structures simulate the structures observed using synchrotron micro tomography and Scanning Electron Microscope imaging. The modeling accounts for the influence of pore fluid and illustrates how grain/pore heterogeneity under dry and saturated states affects stress wave and grain damage behavior. The simulations show characteristics of the phenomena observed in recovery experiments. An increase in grain damage coincides with an increase in stress level and pulse duration. The grains in dry samples are extremely and irregularly fragmented with extensively reduced porosity. Less grain damage and higher porosity is observed in the saturated samples. The influence of pore fluid mitigates the interaction between grains, thus reducing fragmentation damage. This modeling approach in concert with experiments offers a unique way to understand dynamic compaction of brittle porous materials.
\end{abstract}

\section{INTRODUCTION}

We use numerical simulations based on a hybrid technique combining Smooth Particle Hydrodynamics (SPH) and the Discrete Element Method (DEM) to model several impact experiments. The SPH method, originally formulated for the treatment of astrophysical problems (1), has been used over the past decade to model solid mechanics problems such as bombcase fragmentation (2), impact events (3), and brittle fracture $(4,5)$. DEM originated with the work of Cundall (6) for applications to rock mechanics and granular assemblies. The DEM method consists of bonded polyhedra that models elastic behavoir of continua and is ideally suited for the simulation of brittle fracturing processes where explicit modeling of crack patterns is desired. By combining SPH with DEM, we are able to take advantage of the strengths of each.

The experiments were designed to characterize the consequences of shock loading Berea sandstone under various conditions including dry, water saturated, and water-pressurized. The motivation for such experiments comes in part from applications to oil well completion technology used by industry. Shaped charges are used to perforate the well casing and reservoir rock in order to connect the well to the reservoir. During the process of completion the perforator jet imposes a stress wave on the rock causing damage to the grains and pores, often reducing the productivity of the completion.

By combining a modeling effort with the experimental results a better understanding of the physical processes involved will develop, and an avenue for scaling physical behavior at the mesoscale to larger scales that require phenomenological models will follow.

The experimental samples were subjected to shock loads ranging from roughly $2 \mathrm{GPa}$ to $9.8 \mathrm{GPa}$ and are either $1 \mu \mathrm{s}$ or $2 \mu \mathrm{s}$ in duration. A diagram showing the experimental setup is shown in Fig. 1. Scanning Electron Microscope (SEM) imaging of the recovered samples has provided a database for assessing visual and statistical differences between areas of a given sample, and between samples exposed to differing experimental parameters. More details of the experiments and recovered samples are reported in this volume (7). Modeling of several experimental conditions was performed on limited domains, and reveals qualitative results of fragmentation and damage similar to that observed in the recovered samples. 


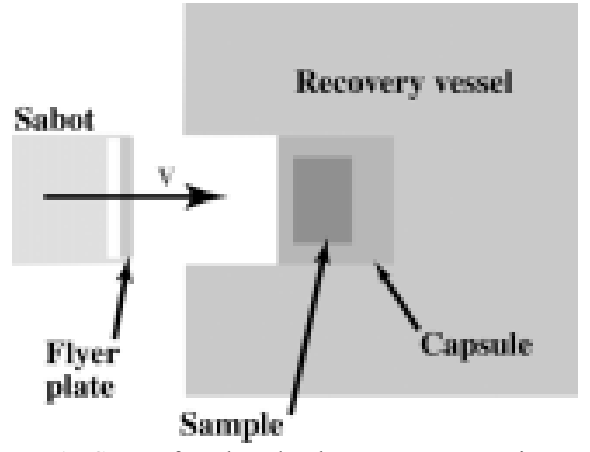

FIGURE 1. Setup for the shock recovery experiments. The diameter of the sample is $22.4 \mathrm{~mm}$. The simulations are limited to the flyer, capsule, and sample and are on a much smaller scale.

The results presented here show that heterogeneity at the grain scale of the sandstone affects stress wave behavior and the damage imparted to the material. We employ the SPH/DEM SPHINX code (8) to model this heterogeneous behavior. Our calculations for two-dimensional representations of grain structure examine the influence of pore space treated as a void or as fluid filled. Although the simulations are twodimensional the results reflect, in a qualitative manner, the complex phenomenology associated with the experiments. Simulation of well-defined plate impact loading captures the effect of stress intensity on the damage observed in samples of Berea sandstone recovered from the gas-gun experiments.

\section{SIMULATION OF EXPERIMENTAL CONDITIONS}

SPH is a Lagrangian, meshless method in which material elements are represented by mass points, known as particles, and are governed by the continuum equations of motion and constitutive models. Particles carry local values of position and velocity, with values of density and deformation rate obtained through weighted difference equations involving neighboring particles. Stress for each particle is obtained from specific constitutive representations, while energy and acceleration are updated from the balance equations. Excellent reviews of the SPH method $(9,10)$ discuss some of the strengths and limitations of SPH.

DEM has evolved to accommodate various applications (11). The method used here consists of assemblies of polyhedra whose interaction is an alternation between momentum balance and a force-displacement law. The force-displacement law is used to find contact forces between particles from their displacements resulting from the momentum balance. Material properties specified by coefficients in the displacement law cause assemblages of DEM particles to behave equivalently to an elastic continuum. In this context, DEM seems very useful for modeling explicit brittle fracture behavior.

\section{GRAIN and PORE STRUCTURE}

Representations of a heterogeneous grain structure for impact loading simulations are created to be similar to images of reservoir rock. We are at the moment relying on synthetic grain structures following (12). Images of Berea sandstone are obtained using either SEM (Fig. 2a) or X-ray Computed Micro Tomography (XCMT) (Fig. 2b). The grains of quartz are the predominant gray value in the images, the pores are colored black, and other minerals and clays appear as values of gray usually different than the value of gray for quartz. (a)

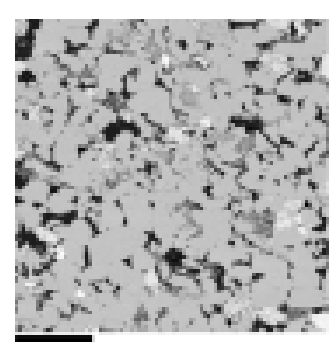

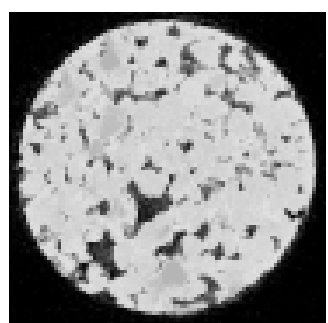

(b)
FIGURE 2. (a) SEM image at $100 \mathrm{x}$ magnification (scale bar represents $500 \mu \mathrm{m}$ ) of undamaged Berea. The dark areas are pores and the lighter grays are grains, or other minerals (calcite, clays, etc.). (b) XCMT image of undamaged Berea. The diameter of the core is approximately $2 \mathrm{~mm}$.

A synthetic grain structure used for modeling is shown in Figure 3. The porosity of the structure is about $23 \%$. The regularity of the boundaries and the grain size distribution can be varied. The pores can be dry, or fluid filled, or filled with any other material. By varying the regularity of the grains and comparing the resulting simulations it was found that angular-boundary assemblies of grains result in more fragmentation compared to smooth-boundary assemblies of grains. 


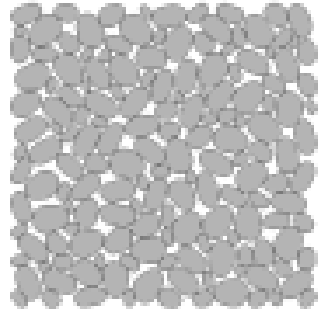

(a)

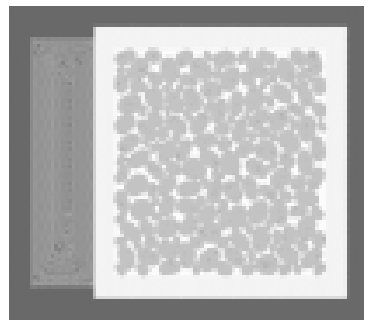

(b)
FIGURE 3. Synthetic grain structure, (a), used for the simulations. This realization contains grains that have generally smoother boundaries than observed grains. The configuration for the simulation of the experiment is shown in (b). The plate impacts the grain-filled box from the left.

Figure 4 shows the axial velocity structure of an SPH/DEM simulation of grains with dry (Fig. 4a) and water-filled pores (Fig. 4b) at a time of 0.4 $\mu$ sec after the initial impact of the plate. The water is modeled using SPH particles having a density of $1 \mathrm{~g} \mathrm{~cm}^{-3}$, whereas the quartz grains are modeled using clusters of DEM triangles having a density of $2.65 \mathrm{~g} \mathrm{~cm}^{-3}$ and coefficients that constitute elastic constants, bulk modulus of $53.7 \mathrm{GPa}$ and shear modulus of $3.0 \mathrm{GPa}$. The aluminum plate, having a velocity of $0.87 \mathrm{~km} / \mathrm{s}$ and density of $2.7 \mathrm{~g} \mathrm{~cm}^{-3}$, can be modeled using either SPH or DEM. The aluminum container is modeled using SPH. Note that the front has propagated less in the dry than in the wet media, indicating that there is more dissipation in the dry grain structure. The velocity structure behind the front is more uniform in the wet case due to the closer match in impedance between the pores and grains relative to the dry case.

Figure 5 shows the crack distribution in the dry (Fig. 5a) and water-filled (Fig. 5b) sample simulated by the DEM portion of the calculation. The cracks are shown at $1.7 \mu$ s after the initial impact. There is much greater compaction and fragmentation evident in the dry sample than in the saturated sample. The increased mass and pore pressure of the water provides a cushioning and mitigates the grain-grain interactions that otherwise result in the increased damage observed in the dry samples.

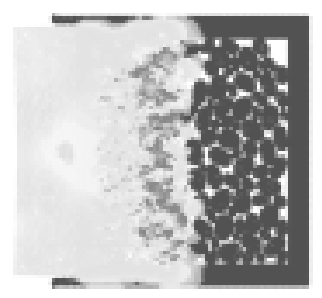

(a) (b)

FIGURE 4. Axial velocity field from an SPH/DEM simulation of a small portion of a dry (a) and wet (b) grain structure at 0.4 $\mu$ s after the impact of the plate from the left. Black and white represent zero velocity, while shades of gray from light to dark represent increasing axial velocity to the right.

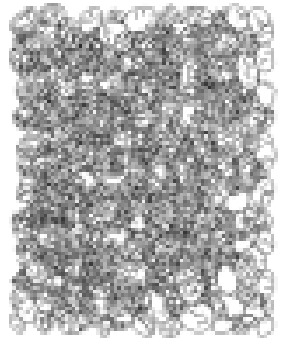

(a)

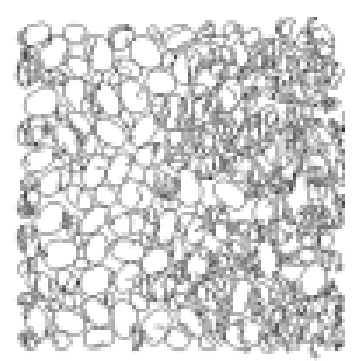

(b)
FIGURE 5. Crack structures (damage) in the dry (a) and wet (b) sample as simulated by SPH/DEM at $1.7 \mu$ s after the initial impact of the flyer plate (not shown) from the left.

Using the recovered samples from the gas-gun impact experiments, we can compare the simulations to SEM images of portions of the sample. The cylindrical samples were cut in half along a plane parallel to the axis of the cylinder, exposing an internal face of the sample similar to the diagram in Fig. 1. Figure 6 shows processed images of recovered samples from dry and watersaturated impact experiments. The segmentation represents the character of the crack patterns. First, voids are outlined (interiors become white), then a threshold is applied to make all grain fragments white, and finally, the image is skeletonized to produce the structure in Figure 6. The result is qualitatively comparable to those produced by the simulation shown in Figure 5. 


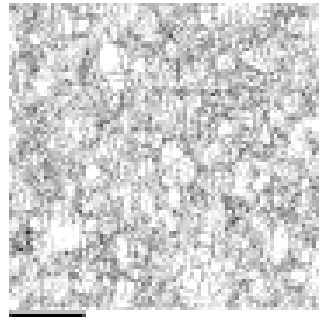

(a)

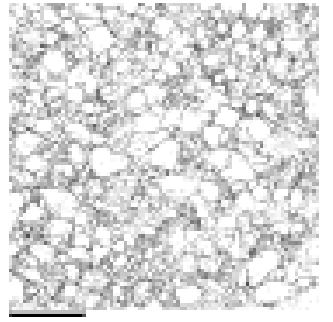

(b)
FIGURE 6. Binary versions of SEM images of the dry (a) and wet (b) recovered samples. The original gray-scale image has been processed to enhance the crack and boundary information.

\section{CONCLUSIONS}

We have demonstrated the use of a combined Smooth Particle Hydrodynamics and Discrete Element Method (SPH/DEM) computational method as a way to model the dynamics of grain-tograin and grain-to-pore interactions on the grain scale. We have used the method to simulate the damage resulting from stress wave loading and compared the results to gas-gun impact experiments using Berea sandstone. Simulations of a plate impact configuration show the influence of explicit heterogeneity for void (dry) pore space and fluid filled pores. Qualitative agreement is obtained for simulated damage with the damage observed on the recovered samples of Berea sandstone from the impact experiments. The significant increase in fragmentation and compaction of the dry sample compared to the water-saturated sample is achieved in the simulation. Although these grain-scale simulations are two-dimensional, they capture the essence of how damage to the rock fabric can occur from perforation-type stress wave loading. The simulations illustrate the complexity of how heterogeneity affects stress wave behavior which in turns affects damage evolution. Increasing the size of the computational domain as parallel implementation of the code is completed will provide enhanced simulation capabilities. Exercising these grain-scale simulations over a wide range of geophysical parameters on large domains will provide information necessary for the development of continuum models.

\section{REFERENCES}

1. Gingold, R. A., and J. J. Monaghan, "Smoothed Particle Hydrodynamics: Theory and Application to Non-spherical Stars", Mon. Not. Roy. Astr. Soc. 181, 373-389, 1977.

2. Randles, P. W., T. C. Carney, and L. D. Libersky, "Continuum Dynamical Simulations of Bomb Fragmentation", Pro. 15th Int. Symp. Ballistics, Jersualem, Israel, May 21-24, 1995.

3. Stellingwerf, R. F. and C. A. Wingate, "Impact Modeling with Smooth Particle Hydrodynamics", Los Alamos National Laboratory Document LAUR-92-1981, 1992.

4. Mandell, D. A. and C. A. Wingate, "Prediction of Material Strength and Fracture of Glass Using the SPHINX Smooth Particle Hydrodynamic Code", Los Alamos Technical Report LA-12830.

5. Mandell, D. A., C. A. Wingate, "Numerical simulations of glass impacts using Smooth Particle Hydrodynamics", APS Topical Conference on Shock Compression in Condensed Matter, Seattle, WA, 1995.

6. Cundall, P. A. and O. D. L. Strack, "A discrete numerical model for granular assemblies", Geotechnique, 29, 47-65, 1979.

7. Hiltl, M., R. P. Swift, C. R. Hagelberg, T. C. Carney, and W. J. Nellis, "Shock-recovery experiments of sandstone under dry and water-saturated conditions", in Shock Compression in Condensed Matter-1999 edited by M. D. Furnish, AIP Conference Proceedings, this volume.

8. Wingate, C. A. et. al., "SPHINX Manual, Version 11.0", Los Alamos National Laboratory Document, LA-13436-M, (1998).

9. Fulk, D. A. "A Numerical Analysis of Smoothed Particle Hydrodynamics", Thesis, Air Force Institute of Technology, 1994.

10. Swegle, J. W., D. L. Hicks, and S. W. Attaway, "Smoothed Particle Hydrodynamics Stability Analysis", J. Comp. Phys. 116, 123-134, 1995.

11. Williams, John R. and Graham G. W. Mustoe, editors. Proceedings of the $2^{\text {nd }}$ International Conference on Discrete Element Methods (DEM). IESL Publications, Dept. of Civil and Environmental Engineering, Massachusetts Institute of Technology, 1993.

12. Tacher, L., P. Perrochet, and A. Parriaux, "Generation of Granular Media", Transport in Porous Media, 26, 99-107, 1997 